\title{
AGTUALIDAD: Recomendaciones de 2013 Sobre Terapia de Remplazo Hormonal. Sociedad Británica de Menopausia y del Cuidado de la Salud de la Mujer.
}

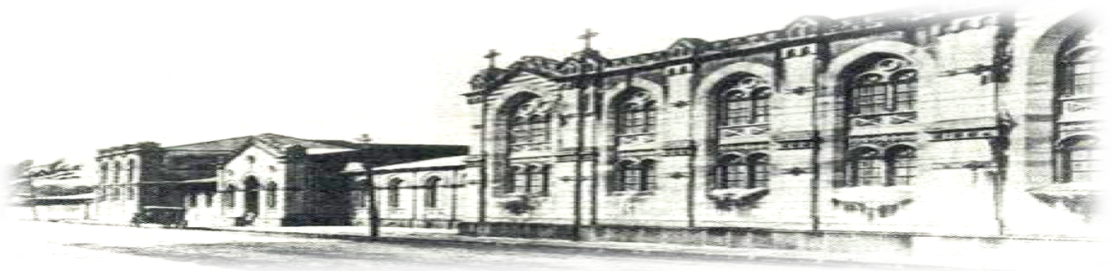

Hospital San quan de Dios, San José. Costa Rica. Fundado en 1845

Recibido: $\quad 22 / 07 / 2013$

Aceptado: $\quad 14 / 08 / 2013$

HSJD

Elena Chaves Goicuria ${ }^{1}$

Ibrahim Barguil Meza ${ }^{2}$

${ }^{1}$ Médica General. Correo electrónico: elechag@gmail.com

${ }^{2}$ Especialista en Medicina Interna. Jefe del Servicio de Medicina Interna HSJD. Profesor de la Cátedra de Medicina Interna, UCR-HSJD. Profesor del posgrado de Medicina Interna UCR- CENDEISSS. Correo electrónico: ibarguil@,ccss.sa.cr

TRH y Síntomas Vasomotores

- El estrógeno continua siendo un tratamiento más eficaz comparado con el placebo.

- Se ha comprobado una mejora en el estado de animo y en síntomas depresivos al inicio del tratamiento.

- Mejora la función sexual en mujeres con dispareunia secundaria a atrofia vaginal.

- Reduce el riesgo en la recurrencia de infecciones urinarias.

- El estrógeno vaginal se puede utilizar a largo plazo en dosis bajas, ya que la absorción sistémica es menor y no es necesario administrarlo junto con progesterona.

- El principal factor que disminuye la continuidad al tratamiento con Terapia de Reemplazo Hormonal (TRH) es la falta de tolerancia hacia los progestágenos.
TRH y Osteoporosis

- Mujeres sintomáticas, menores de 60 años y mujeres con insuficiencia prematura de ovario deben recibir como primera opción la TRH para prevenir osteoporosis.

- En las pacientes mayores de 60 años se demostró que el uso de TRH no es recomendado.

TRH y Efectos Cardiovasculares

- Las mujeres mayores de 60 años que inician con TRH por primera vez, ocurre lo que se conoce como un "daño temprano" (efectos cardiovasculares), por lo que es necesario iniciar con la dosis efectiva más baja, para evitar estos efectos adversos. 


\section{TRH y Cáncer}

\section{Cáncer de Mama}

- En el estudio WHI sobre estrógeno y progesterona se detecto un incremento leve en el riesgo de padecer cáncer de seno, después de 5 años con tratamiento.

- En el estudio WHI de solo estrógeno, se observo un pequeño decremento en el riesgo de padecer cáncer de seno.

\section{Cáncer de Ovario}

- En un ensayo controlado con placebo del estudio WHI, se investigó la relación entre TRH y cáncer de ovario, se concluyó que no existía incremento alguno en el riesgo.

- Un reporte del Centro Nacional Danés del Cáncer, revelo un leve aumento en la incidencia de cáncer de ovario, después de 8 años con terapia sola o combinada.

\section{Cáncer de endometrio}

- El uso de estrógeno, aumenta la incidencia de cáncer de endometrio, este riesgo se evita al agregar progesterona.

- Después de 5 años con TRH combinada existe un leve incremento en el riesgo de cáncer de endometrio, sin embargo el régimen continuo combinado tiene un riesgo mucho menor, si se compara a una población menopaúsica sin tratamiento.

\section{TRH después del Cáncer}

\section{Cáncer de endometrio}

- Los sarcomas del endometrio son tumores sensibles al estrógeno, por lo tanto son una contraindicación en el uso de la TRH.

\section{Cáncer de Ovario}

- En el tumor de células de la granulosa se debe de evitar la TRH, ya que no existen estudios que lo respalden.

\section{Cáncer de Cérvix}

- La TRH no esta contraindicada después de un adenocarcinoma de cérvix y de un carcinoma de células escamosas.

\section{Cáncer de vulva}

- El estrógeno sin importar la vía de administración se puede utilizar después de un cáncer de vulva, ya que no existe evidencia de recurrencia.

\section{TRH y Trombo-embolismo Venoso}

- El uso de TRH incrementa el riesgo 2 a 4 veces más, durante el primer año de tratamiento.

- El riesgo de trombo-embolismo venoso es mucho mayor si existen comorbilidades.

- El riesgo se asocia al uso de TRH oral y no a la vía transdérmica. Sin embargo al combinar los parches con progestágenos como el acetato de medroxiprogesterona y los derivados de la norpregnona el riesgo es mayor.

- Las pacientes con riesgo que ameriten tratamiento, se les debe recomendar como mejor opción los parches transdérmicos y en caso de requerir progesterona se debe agregar la forma micronizada o la didrogesterona.

\section{Rutas y Regímenes de la TRH}

- El estrógeno transdérmico y subcutáneo, evita el metabolismo de primer paso por el hígado, por lo tanto no se asocia a un aumento en el riesgo de trombosis venosa.

Alternativas Farmacológicas para síntomas Vasomotores

- La Fluoxetina y la Paroxetina se destacaron en los estudios por aliviar síntomas de la menopausia.

- La Venlafaxina a una dosis de $37.5 \mathrm{mg}$ bid se ha utilizado en sobrevivientes de cáncer.

- La Gabapentina demostró ser eficaz para reducir los calores asociados a la menopausia.

\section{Fitoestrógenos}

- Asociados a una disminución de hasta un $60 \%$ de los síntomas vasomotores, sin embargo los beneficiosos son mayores con el uso de TRH tradicional (disminución de $90-100 \%)$.

\section{Puntos Clave}

- La decisión de iniciar TRH, debe ser tomada por cada mujer, luego de haber recibido información por el profesional en salud, en cuanto a sus ventajas y desventajas. 
- La dosis/régimen y duración de TRH debe ser individualizada, con chequeos anuales.

- Al iniciar TRH, no se debe imponer limites en cuanto al período que se debe utilizar. Si los síntomas persisten, los beneficios de la TRH sobrepasan los riesgos.

- Cuando se prescribe TRH en mujeres menores de 60 años, los resultados obtenidos son más favorables.

- Es primordial que a todas las mujeres con insuficiencia prematura de ovario se les estimule a utilizar TRH, hasta la edad promedio de la menopausia.

- Al utilizar TRH en mujeres mayores de 60 años, se debe iniciar con la dosis más baja preferiblemente por la vía transdérmica.

- Es indispensable que hoy en día, con el aumento en las terapias hormonales, se logre maximizar los beneficios y minimizar los riesgos.

\section{BIBLIOGRAFÍA}

1. British Menopause Society Council. Modernizing the NHS: observations and recommendations from the British Menopause Society. Menopause Int 2011; 17:41-43.

2. Bromberger JT Schott LL Kravitz HM et al. Longitudinal change in reproductive hormones and depressive symptoms across the menopausal transition: results from the study of Womens Health Across the Nation $(S W A N)$. Arch Gen Psychiatry 2010;67: 598-607.

3. De Villiers TJ Pines A Panay N. Updated 2013 International Menopause Society Recommendations on menopausal hormone therapy and preventive strategies for midlife health. Climateric 2013;16:316-337.

4. Maki PM Freeman EW Greendale GA et al. Summary of the National Institute on Aging-sponsored conference on depressive symptoms and cognitive complaints in the menopausal transition. Menopause 2010; 17:815-822.

5. Studd JWW. A guide to the treatment of depression in women by estrogens. Climateric 2011;14: 637-642.

6. Cody JD Richardson $\mathrm{K}$ Moehrer $\mathrm{B}$ et al. Estrogen therapy for urinary incontinence in post-menopausal women. Cochrane Database Syst Rev 2009;4:CD001405.
7. Sturdee DW Panay N. Recommendations for the management of postmenopausal vaginal atrophy. Climateric 2010;13:509522.

8. Calleja-Agius J Muscat-Baron Y Brincat MP. Estrogens and the intervertebral disc. Menopause Int 2009;15:127-130.

9. Calleja-Agius J Brincat MP. Effects of hormone replacement therapy on connective tissue: why is this importat? Best Pract Res Clin Obstet Gynaecol 2009; 23:121.

10. Bednareck PH Jenesn JT. Safety, efficacy and patient acceptability of the contraceptive and non-contraceptive uses of the LNG-IUS. Int J Women Health 2009; $1: 45-58$.

11. Cauley JA Robbins J Chen Z et al. Effects of estrogen plus progestin on risk of fracture and bone mineral density: the Women's Health Initiative randomized trial. JAMA 2003;290:1729-1738.

12. Stevenson JC International Consensus Group on HRT and Regulatory Issues. HRT, osteoporosis and regulatory authorities. Hum Reprod 2006;21:1668-1671.

13. Harman SM Brinton EA Cedars M et al. KEEPS: the Kronos early estrogen prevention study. Climateric 2005;8:3-12.

14. Grodstein F Manson JE Stampfer MJ. Hormone therapy and coronary heart disease: the role of time since menopause and age at hormone initiation. J Women's Health 2006; 15:35-44.

15. Schierbeck LL Rejnmark L Tofteng $\mathrm{Cl}$ et al. Effects of hormone replacement therapy on cardiovascular events in recently postmenopausal women: randomized trial. BMJ 2012;345:e6409.

16. Collaborative Group on Hormonal factor in Breast cáncer. Breast cancer and hormone replacement therapy: collaborative reanalysis of data from 51 epidemiological studies of 52705 women with breast cancer and 108411 women without breast cancer. Lancet 1997;350:1047-1059.

17. Panay N. Commentary regarding recent Million Women Study critique and subsequent publicity. Menopause Int 2012; 18:33-35.

18. Ravdin PM Cronin KA Howlader N et al. The decrease in incidence of breast cancer in the United States. New Engl J Med 2007; 356:1670-1674. 
19. Canonico M Plu-Bureau G Lowe GD et al. Hormone replacement therapy and risk of venous thromboembolism in postmenopausal women: systematic review and meta-analysis. BMJ 2008;336:12271231.

20. Scarabin PY Oger E Plu-Bureau G. Differential association of oral and transdermal estrogen replacement therapy with venous thromboembolism risk. Lancet 2003;362:428-432.

21. Panay N Ylikorkala O Archer DF et al. Ultra low dose estradiol and norethisterone acetate: effective menopausal symptom relief. Climateric 2007;10:120-131.

22. Nelson HD Vesco KK Haney E et al. Non hormonal therapies for menopausal hot flashes: systematic review and metaanalysis. JAMA 2006;295:2057-2071.

23. Sassarini J Lumsden MA. Hot flushes: are there effective alternatives to estrogen? Menopause Int 2010;16:81-88.

24. Fenton A Panay N. The Women's Health Initiative- a decade of progress. Climateric 2012;15:205.

25. North American Menopause Society. The 2012 hormone therapy position statement of The North American Menopause Society. Menopause 2012;19:257-271. 